\title{
Image Texture Analysis and Gas Sensor Array Studies Applied to Vanilla Encapsulation by Octenyl Succinic Anhydride Starches
}

\author{
Silvio D. Rodríguez ${ }^{1}$, Tom F. Wilderjans ${ }^{2}$, Natalia Sosa $^{3} \&$ Delia L. Bernik ${ }^{1}$ \\ ${ }^{1}$ Instituto de Química Física de Materiales, Ambiente y Energía (INQUIMAE), Facultad de Ciencias Exactas y \\ Naturales, Universidad de Buenos Aires, Buenos Aires, Argentina \\ ${ }^{2}$ Methodology of Educational Sciences Research Group, Faculty of Psychology and Educational Sciences, KU \\ Leuven, Leuven, Belgium \\ ${ }^{3}$ Departamentos de Industrias y de Química Orgánica, Facultad de Ciencias Exactas y Naturales-Universidad de \\ Buenos Aires, Buenos Aires, Argentina \\ Correspondence: Delia L. Bernik, Instituto de Química Física de Materiales, Ambiente y Energía (INQUIMAE), \\ Facultad de Ciencias Exactas y Naturales-Universidad de Buenos Aires, Ciudad Universitaria, Buenos Aires \\ C1428EGA, Argentina. Tel: 54-11-4576-3358. E-mail: dbernik@qi.fcen.uba.ar
}

\author{
Received: November 15, 2012 Accepted: January 19, 2013 Online Published: February 27, 2013 \\ doi:10.5539/jfr.v2n2p36 \\ URL: http://dx.doi.org/10.5539/jfr.v2n2p36
}

\begin{abstract}
Native starch derivatization with octenyl succinic anhydride (OSA) is a chemical modification designed to enhance flavor microencapsulation performance. Hi Cap 100 and Capsul are two OSA starches derived from waxy maize base, which are especially suited for encapsulation processes. This work performs for the first time the encapsulation of vanilla extract with Capsul and Hi Cap 100 using both spray and freeze drying procedures. The encapsulation efficiency was studied correlating the starch texture with the aroma retention. Texture analysis was accomplished by means of grey level co-occurrence matrix feature extraction (GLCM), yielding image parameters that clearly differ in function of the type of starch and the drying method used for the encapsulation of the flavor. In parallel, the data recorded with a gas sensor array (e-nose) and analyzed by unsupervised multivariate methods allowed to follow up the evolution of the aroma through the whole process. The joint analysis of the GLCM and sensor array recorded data indicates that Capsul shows a higher capacity for vanilla encapsulation than Hi Cap 100. In addition, the obtained converging information from GLCM and e-nose data clearly indicates that particle texture and aroma encapsulation are connected.
\end{abstract}

Keywords: gas sensor array, OSA starches, GLCM texture analysis, spray and freeze drying, vanilla, chemometric analysis, cluster and component analysis

\section{Introduction}

Chemical modification of native starches to enhance their performance in microencapsulation, a widely used technique in the food industry, has been a topic of interest for many years. For example, starch derivatization with octenyl succinic anhydride (OSA) disrupts the hydrogen bonding and reduces retrogradation of the starch, while increasing its hydrophobicity. In this way, OSA modified starches have been used to stabilize emulsions and to encapsulate sensitive ingredients (Bhosale \& Singhal, 2006; Cova et al., 2010). Hi Cap 100 and Capsul are two commercially available hydrolyzed OSA starches derived from waxy maize base, which are especially suited for encapsulation processes. The only difference in structure between Hi Cap 100 and Capsul is that Hi Cap 100 is a straight chain starch, whereas Capsul is a branched chain starch (Ratchahat et al., 2010). In Figure 1, the general chemical structure of OSA modified starches is depicted. 


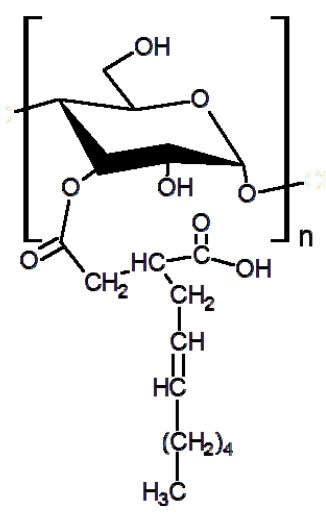

Figure 1. Schematical representation of the general chemical structure of OSA modified starches

Engineering aspects of microencapsulation such as the choice of the drying process and sample components will determine the quality of the obtained powder, its topology, and the ultimate swelling of the final product. The most common industrial method is the spray drying process, which is based on the atomization of a liquid sample into a hot air stream (Gharsallaoui et al., 2007). Although the very short residence time and the relatively low temperatures to which the materials are exposed to, does little to induce oxidation in starch materials, the temperatures involved in this process may damage other components that are very sensitive to oxidation. To avoid this, a less harmful procedure such as freeze drying may be used, although it is more expensive for mass production (Anwar \& Kunz, 2011). For example, in the pharmaceutical industry in which vanilla is used both as an antioxidant and as a masking agent (Tai et al., 2011), the use of freeze-drying is almost mandatory in spite of its higher cost. As such, lowering production costs is a challenging and important topic of ongoing research (Pisano et al., 2011; Lopez-Quiroga et al., 2012).

In this work two main aspects of vanilla encapsulation are studied which may be functionally linked: the final texture of the product (i.e., dried powder) and the efficiency of flavor encapsulation and release. To this end, different proportions of vanilla extracts are added to the respective starches (i.e., $1 \%, 2.5 \%$, and $5 \%$ in weight). The characteristics of the obtained dried powders (i.e., particle size, shape, and texture) are determined by means of an exhaustive inspection of scanning electron microscopy (SEM) pictures, and analyzed with a statistical approach for image texture studies, which is called the grey level co-occurrence matrix (GLCM) technique. In this technique, various textural features are extracted from a matrix that is derived from the information that is contained in the pixels (i.e. the basic components of an image). These textural features are then used to characterize the texture of the product. The advantages of using this relatively new tool, which implies a new trend in image analysis for evaluating food quality, were discussed in the excellent review published by Zheng et al. (2006), and this methodology was recently applied in several cases (Yu et al., 2012; Tournier et al., 2012; Kamruzzaman, 2012).

The second aspect pertains to flavor encapsulation and release from the samples. To study this, sensor arrays, called electronic noses (e-noses), are used; e-noses are devices composed of an ensemble of gas sensors which mimic the sense of smell. In numerous studies, these devices have been demonstrated to be useful for evaluating, both from a qualitative and a semi-quantitative point of view, the release of aroma as a whole from different samples (Rodríguez et al., 2010).

The aim of this paper is to examine how, throughout the procedure of flavor encapsulation, the interaction between the starch and the flavor develops along each step of the drying process (i.e., spray versus freeze drying) and how this interaction is influenced by the molecular structure of the modified starches that are used (i.e., Capsul versus Hi Cap 100). The work intends to demonstrate that the combination of GLCM image texture and e-nose measurements is useful for the evaluation of the encapsulation ability and the final recovery of the aroma when using OSA modified starches. The performance of different drying processes, such as freezing and spray drying is also evaluated. Through the paper, a careful chemometric analysis is adopted in order to ensure a correct interpretation of the results, demonstrating the importance of the adopted multivariate techniques to interpret the data (Peris \& Escuder-Gilabert, 2009; Skov \& Bro, 2005). 


\section{Materials and Methods}

\subsection{Materials}

Hi Cap 100 and Capsul (from National Starch and Chemical) starches were kindly gifted by Gelfix S.A. (Argentina). Vanilla extract was purchased from El Castillo S. A. (Argentina). This vanilla extract has $5 \%$ of ethanol content (certified by ANMAT: Administración Nacional de Medicamentos, Alimentos y Tecnología Médica, Argentina).

\subsection{Experimental Method}

\subsubsection{Preparation of Starch Dispersions in Water with Different Proportions of Vanilla Extract (S1 Samples)}

To see above which threshold concentration the addition of vanilla extracts results in a modification of the original starch structure, samples with different flavor to starch ratios were prepared. $15 \mathrm{~g}$ of the respective OSA starch was dispersed into $38.75 \mathrm{ml}$ of Milli-Q water (with a starch weight percentage of $30 \%$ in all cases) with a mechanical stirrer at $1000 \mathrm{rpm}$ within a water bath at $40^{\circ} \mathrm{C}$ for 40 minutes. Immediately, different aliquots of vanilla extract (VE) were added to the slurry in order to obtain samples with $1 \%, 2.5 \%$, and $5 \%$ of vanilla extract; samples with $0 \%$ of vanilla extract were used as control samples. As the vanilla extract has a $5 \%$ ethanol content (see Section 2.1), the percentage of ethanol that was initially incorporated in the sample (coming from the aliquot of the added vanilla extract) was as low as $0.05 \%, 0.13 \%$, and $0.26 \%$ for the $1 \%, 2.5 \%$, and $5 \%$-samples, respectively. The obtained samples are denoted as "S1 samples" (i.e., first suspension of the starch with flavor prior to any drying process).

\subsubsection{Drying Process (S2 Samples)}

\subsubsection{Spray Drying}

Each S1 sample was spray-dried using a laboratory scale device, Mini Spray Dryer Büchi B290 (Flawil, Switzerland). The operational conditions of the drying process were: inlet air temperature of $170 \pm 3^{\circ} \mathrm{C}$, outlet air temperature of $90 \pm 3^{\circ} \mathrm{C}$, flow rate $8 \mathrm{~mL} / \mathrm{min}$, air pressure $3.2 \mathrm{bar}$, nozzle diameter $1.5 \mathrm{~mm}$.

\subsubsection{Freeze Drying}

Each S1 sample was placed into a Petri dish and frozen at $-18^{\circ} \mathrm{C}$ in a conventional freezer. Next, samples were dried for 24 hours into a Christ ALPHA 1-2 LD plus freeze dryer (i.e., 12 hours in main drying mode at 0.63 mbar and 12 hours in final drying mode at 0.023 mbar).

In both cases, the obtained powders were collected into sealed polyvinylidene chloride bags and stored at about $18^{\circ} \mathrm{C}$.

\subsubsection{Re-Suspension of Dried Powders in Water (S3 Samples)}

The dried powders (i.e., S2 samples) were mixed with Milli-Q water until complete homogenization, resulting in the same starch concentration as for the S1 samples. The obtained samples are called S3 samples.

\subsubsection{Weight Percentage of the Retained Vanilla Extract by Encapsulation in S2 Powders}

To extract vanilla from S2 powders, $0.1 \mathrm{~g}$ of each powder was extracted with $10 \mathrm{ml}$ of ethanol in a sealed vial during 90 min under magnetic stirring. After the extraction, the supernatant was filtered with 0.44 um pore size and the absorbance was measured at a wavelength of $277 \mathrm{~nm}$ with a T60 PG Instruments spectrophotometer. In order to determine the required total extraction time $(90 \mathrm{~min})$, prior tests were conducted by taking small aliquots of supernatant from the extraction vial at different times until the UV absorbance of two consecutive spectra reached its plateau (i.e., no more vanillin was extracted).

\subsubsection{Scanning Electron Microscopy (SEM) to Study Powder Characteristics}

Few milligrams of the S2 samples were placed on a SEM aluminium holder, which was supported on conductive carbon tape. The samples were coated with gold by using a sputter coater (Cressington Scientific Instruments 108) and examined at different magnifications using a Zeiss Supra 40 Gemini and an acceleration potential of $5 \mathrm{kV}$.

2.2.6 Image Texture and Statistical Texture Analysis by the Grey Level Co-Occurrence Matrix Technique

A grey-scale image, as obtained with microscopy, can be represented as a matrix that contains in every cell a grey value that may range from 0 to 255. From this image, after defining the step size or distance $d$ and the angle $\theta\left(0^{\circ}, 45^{\circ}, 90^{\circ}\right.$, and/or $\left.135^{\circ}\right)$, a grey level co-occurrence matrix (GLCM) may be obtained. The resulting GLCM contains 256 rows and columns, with each column and row representing a grey tone between 0 and 255 , and each cell of the GLCM containing the frequency of finding a pair of values (given $d$ and $\theta$ ) with those grey tones indicated by the associated row and column. 
In order to study the texture of the obtained dried starch samples, five different representative features are extracted from the GLCM matrix. First, the angular second moment (ASM), which is defined as a measure of the homogeneity of the image: an image with a smooth surface (i.e. few grey transitions) will give a lower value of ASM than an image of a sample with a larger roughness (inhomogeneous image). Second, the contrast parameter (CT), which represents the amount of local variations given by differences in the grey values in the image: smooth surfaces will result in lower CT values than rough surfaces. Third, the correlation value (CR), which is a measure of grey tone linear dependencies in the image depending on the direction of the measure (different $\theta \mathrm{s}$ ): an image can have a low CR value in one direction and a higher CR value in another direction. Fourth, the inverse difference moment value (IDM), which, similar to ASM, quantifies the homogeneity of the image, however, using a different equation. Finally, the entropy parameter (ET), which is a measure that is inversely related to the order given by the grey tones in the image: a smooth surface will show a lower value of ET than a rough surface (Arzate-Vázquez et al., 2012; Zheng et al., 2006).

To compare the texture, frames of $200 \times 200$ pixels from SEM images of S2 samples (8000× in all the cases) of Capsul and Hi Cap 100 with $0 \%$ and $5 \%$ of vanilla extract (VE) were used. Control samples were selected from perfectly smooth spherical particles, randomly chosen among both starches with and without VE. The ImageJ software was used to perform the GLCM features extraction.

\subsubsection{E-Nose Measurements to Study the Flavor Pattern Released by the Samples}

A sensor array (e-nose) prototype developed in our laboratory was used. The device is composed of twelve commercial gas sensors based on polycrystalline tin dioxide (Figaro, Inc); more technical information can be found in Rodríguez et al. (2010). The different stages S1, S2, and S3 of each sample for both drying processes (i.e., spray and freeze) were analyzed with the e-nose device.

For S1 samples, an aliquot of $5 \mathrm{ml}$ of the slurry was put in a Petri dish and placed into the e-nose chamber, and the aroma was registered. For S2 samples (consisting of the whole amount of dried powder obtained from the S1 samples after drying), the Petri dish with the powder was put into the e-nose chamber and the aroma of the headspace was measured. The S3 samples were obtained by re-suspension of the S2 powders in $5 \mathrm{ml}$ of water. As a consequence, the initial starch concentration is recovered. The S3 samples were placed again in a Petri dish in the sensor chamber and the aroma release was measured in the same way than for the S1 and S2 samples, as described in the beginning of this paragraph. In all cases, the e-nose baseline was resettled by pumping pure air and the temperature of the chamber was controlled at $20^{\circ} \mathrm{C}$ by a circulating water bath. Measurements were performed by triplicate, until sensor's signals reached a steady response, which took about 20 minutes (i.e., 1,200 seconds). Note that e-nose measurements were only performed for samples with a $2.5 \%$ vanilla to starch weight ratio, because this proportion allows visualizing the evolution of the changes in odour intensities without a very fast saturation of the sensors readings.

\subsection{Data Analysis}

The raw sensor's recordings were used for data analysis. Normalization of the data was not used in any case because in this study the signal intensity is a key variable along the encapsulation/re-suspension process.

\subsubsection{Two-Way Component Methods: Principal Component Analysis/Unfolded Principal Component Analysis}

Principal Component Analysis (PCA) is a well-known method for determining similarities among the objects in a two-way data set (e.g., samples by sensors). To this end, the relative locations of the samples on the most important principal components in a two- or three-dimensional plot are compared (Jolliffe, 2002). PCA on the covariance matrix was performed using the Infostat/p2011 software.

When having a three-way data set (e.g., samples by sensors by time points), unfolded PCA (also called multi-way PCA, Nomikos \& MacGregor, 1994) may be performed, which consists of applying PCA to the two-way matrix that is obtained by unfolding the three-way matrix by concatenating the different slices of the three-way matrix horizontally (Kiers, 2000).

\subsubsection{Cluster Analysis (K-Means Method)}

In Cluster Analysis (CA), a set of objects (in this case each recorded data from the e-nose measurements of the samples) is assigned to a number of groups (i.e., clusters), specified by the user, in such a way that objects belonging to the same cluster are more similar to each other than objects belonging to different clusters (Johnson $\&$ Wichern, 2002). We performed a $K$-means analysis, using the Infostat/p2011 software. 


\subsubsection{Three-Way Component Methods: PARAFAC- and PARAFAC2-Analysis}

To analyze the three-way data set which contains the sensor's signals for each sample at different time points, one may use PARAFAC (Harshman, 1970; Carroll \& Chang, 1970) or PARAFAC2 (Harshman, 1972), which can be conceived as two related generalizations of PCA to three-way data. As in PCA, a limited number of components are obtained and scores for the samples, sensors, and time points on these components can be determined. We performed a PARAFAC- and PARAFAC2-analysis with two components, using the MATLAB N-way toolbox (Andersson \& Bro, 2000).

\subsubsection{Multivariate Analysis of Variance (MANOVA)}

Multivariate ANOVA (MANOVA) is a statistical method that is used to test differences among means of several groups with respect to more than one dependent variable. MANOVA is aimed at examining the differences across multiple dependent variables by simultaneously testing the null hypothesis that the population means of the groups are equal to one another for all the dependent variables under study (Haddi et al., 2011). In this study, MANOVA analysis was performed on the data set presented in Table 1 (note that Table 1 only shows the mean values for each group and for each dependent variable).

\section{Results}

\subsection{Image Texture Analysis: Characteristics of the Powder}

When the texture of the SEM images from freeze-dried S2 samples were analyzed, no measurable differences between the Hi Cap 100 (Figure 2, part a) and Capsul (part b) starches showed up. In particular, both starch types yielded irregular thick sheets, and the addition of the extract did not introduce any noticeable change (pictures not shown).

When using spray drying, on the contrary, clear textural differences were obtained for particles belonging to different starch types. In Figure $2 \mathrm{c}$ and d in which the SEM images of S2 Capsul (c) and Hi Cap 100 (d) are presented, Capsul clearly showed particles with a much rougher surface than Hi Cap 100.
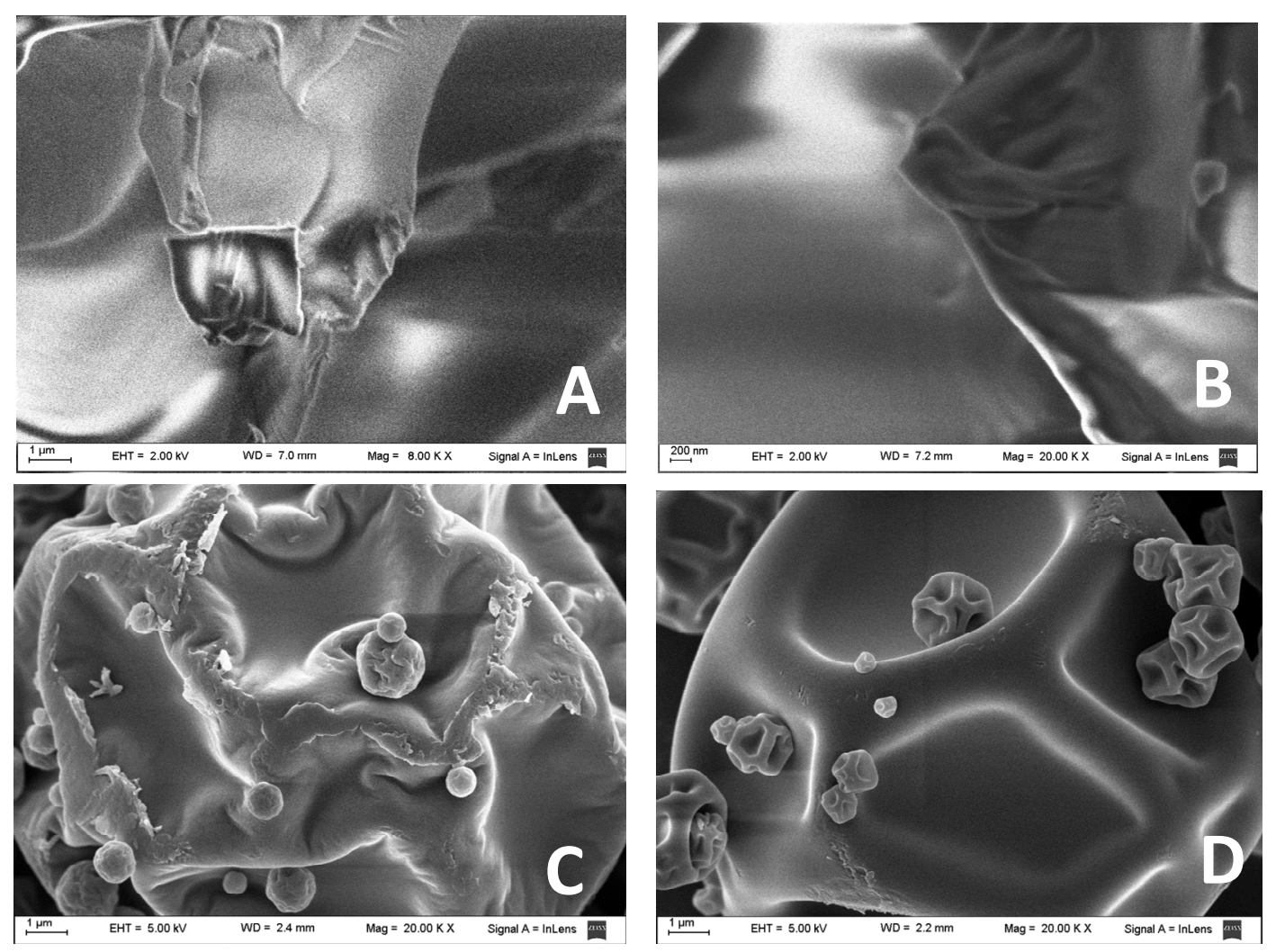

Figure 2. SEM images obtained from S2 samples of freeze (a and b) and spray-dried (c and d) particles of Capsul (a and c) and Hi Cap 100 (b and d) starches, respectively, without Vanilla extract. The surface topography of the dried powders is showed at a magnification of $20000 \times$ 
To confirm these visual differences, texture analysis was performed using GLCM on the spray dried particles (see section 2.2.6). Note that the GLCM textural parameters should be interpreted as follows: larger ASM, CR, and IDM values indicate less roughness, whereas larger CT and ET values indicate more roughness.

In Table 1 , for $d=1, \theta=0^{\circ}$, and $\theta=90^{\circ}$, the average values and their standard deviations for the five textural parameters are displayed as a function of the starch type without and with $5 \%$ of vanilla extract added. Note that similar results were obtained for $\mathrm{d}=5, \theta=0^{\circ}$, and $\theta=90$ (data not shown).

Table 1. Average values and standard deviations of textural feature parameters extracted from the GLCM $(d=1)$ from S2 spray-dried samples of Capsul and Hi Cap 100 (and control) starch samples with (5\%) and without the addition of vanilla essence

\begin{tabular}{cccccccc}
\hline & & \multicolumn{5}{c}{ Textural features } \\
\cline { 4 - 8 } & $\begin{array}{c}\text { Starch } \\
\text { samples* }\end{array}$ & $\begin{array}{c}\text { VE } \\
\text { weight } \%\end{array}$ & $\begin{array}{c}\text { ASM } \\
\left(\times 10^{4}\right)\end{array}$ & CT & $\begin{array}{c}\text { CR } \\
\left(\times 10^{4}\right)\end{array}$ & IDM & ET \\
\hline $\mathrm{d}=1$ & Capsul (4) & $0 \%$ & $2.8 \pm 0.1$ & $205 \pm 23$ & $5.0 \pm 0.2$ & $0.12 \pm 0.01$ & $8.7 \pm 0.1$ \\
$\theta=0^{\circ}$ & Capsul (4) & $5 \%$ & $2.7 \pm 0.2$ & $158 \pm 9$ & $4.3 \pm 0.5$ & $0.13 \pm 0.01$ & $8.7 \pm 0.1$ \\
& Hi Cap (4) & $0 \%$ & $4.0 \pm 0.9$ & $97 \pm 12$ & $5.5 \pm 0.9$ & $0.15 \pm 0.01$ & $8.4 \pm 0.2$ \\
& Hi Cap (4) & $5 \%$ & $3.8 \pm 0.4$ & $93 \pm 6$ & $5.4 \pm 0.2$ & $0.15 \pm 0.01$ & $8.3 \pm 0.2$ \\
& Control (11) & $\#$ & $16.4 \pm 0.8$ & $28 \pm 9$ & $74.7 \pm 55$ & $0.21 \pm 0.02$ & $6.8 \pm 0.5$ \\
\hline $\mathrm{d}=1$ & Capsul (4) & $0 \%$ & $2.1 \pm 0.1$ & $343 \pm 17$ & $4.8 \pm 0.2$ & $0.10 \pm 0.01$ & $8.9 \pm 0.1$ \\
$\theta=90^{\circ}$ & Capsul (4) & $5 \%$ & $2.1 \pm 0.2$ & $271 \pm 36$ & $4.2 \pm 0.5$ & $0.10 \pm 0.01$ & $8.9 \pm 0.1$ \\
& Hi Cap (4) & $0 \%$ & $3.0 \pm 0.7$ & $175 \pm 19$ & $5.5 \pm 0.9$ & $0.11 \pm 0.01$ & $8.6 \pm 0.2$ \\
& Hi Cap (4) & $5 \%$ & $2.9 \pm 0.3$ & $154 \pm 24$ & $5.3 \pm 0.2$ & $0.11 \pm 0.01$ & $8.6 \pm 0.1$ \\
& Control (11) & $\#$ & $13 \pm 7$ & $53 \pm 15$ & $60 \pm 31$ & $0.16 \pm 0.02$ & $7.1 \pm 0.5$ \\
\hline
\end{tabular}

ASM: angular second moment; CT: contrast parameter; CR: correlation value; IDM: inverse difference moment; ET: entropy parameter (for a detailed description of each parameter, see section 2.2.6);

* The number of samples used in the analysis is denoted between parentheses;

\# Samples were randomly chosen among perfectly smooth particles from both starches with and without VE.

From Table 1, it appears that Capsul samples, in comparison to Hi Cap 100 samples which showed less rougher particles, yielded larger CT and ET parameter values and lower ASM, IDM, and CR parameter values; this result is supported by a MANOVA $(F(5,20)=16.7, p<.0001)$. Further, for the five textural parameters, the control samples clearly differed from the Capsul and Hi Cap 100 samples, with a MANOVA yielding the same result $(F(5,20)=78.5, p<.0001)$.

Cluster analysis with two clusters yielded a perfect classification of the samples into control smooth samples, on the one hand, and Capsul and Hi Cap 100 particles, on the other hand. Moreover, when choosing three clusters, the samples were grouped with a success rate of $100 \%$ in Capsul, Hi Cap 100, and smooth control samples.

After the addition of flavor, some changes were observed in the texture parameters for samples from the same starch type, with these changes being the largest for the contrast parameter CT. In particular, CT decreases, which suggests that adding the flavor especially decreases the roughness of the powders/samples. Note that, to a lesser extent, a similar pattern is obtained for the other texture parameters. However, when five clusters were selected in CA (i.e., two starch types with and without VE, and the control smooth samples), the observed differences are not large enough to get the five groups perfectly separated (i.e., $36 \%$ misclassifications).

A final question pertains to the effect of adding flavor on the average value of the particle's size distribution. In order to confirm this, particles were randomly selected from different 1000 $\times$ SEM images and their diameters were measured. Four selected SEM pictures are displayed in Figure 3, showing the change in the size distribution. 

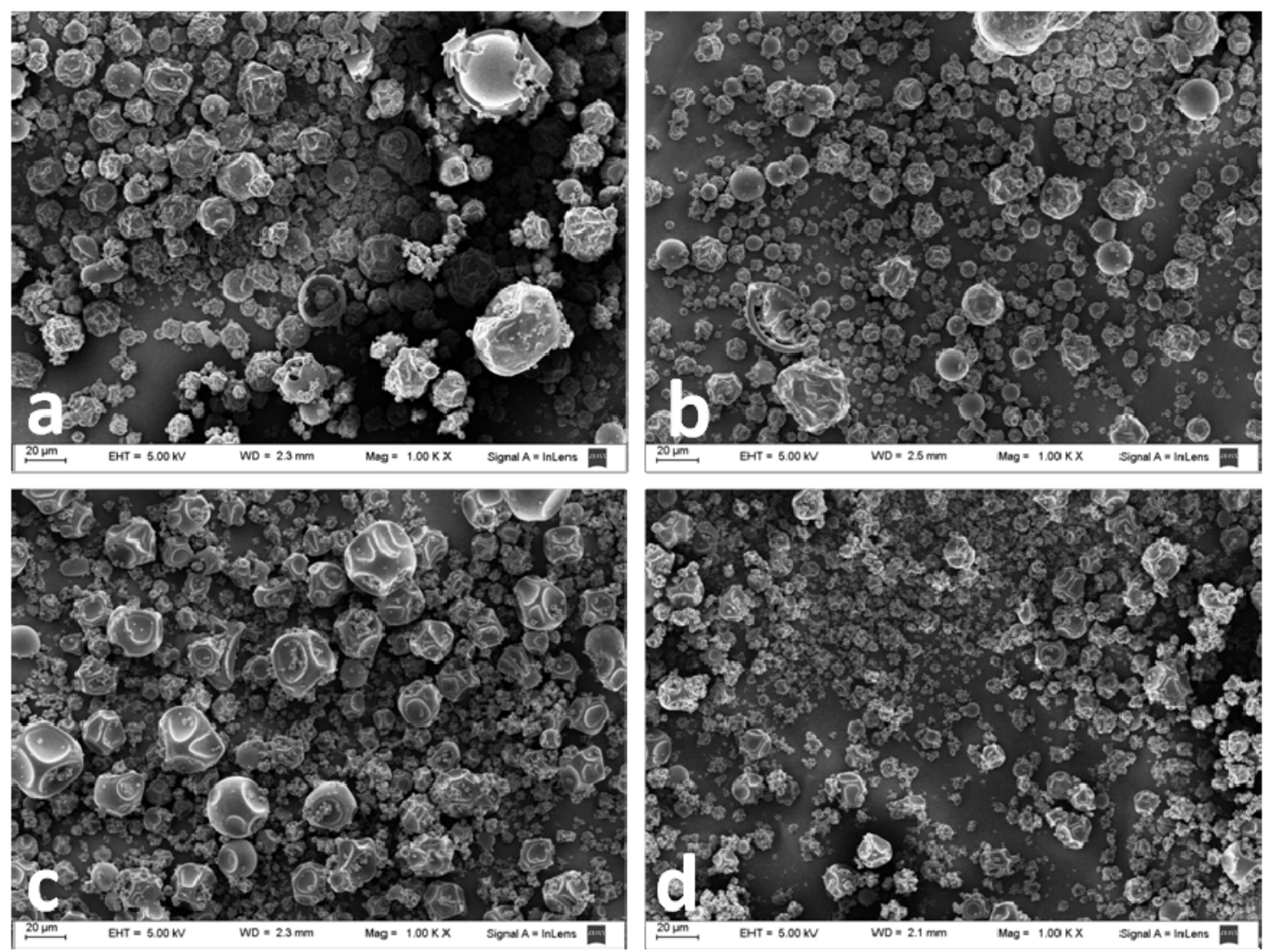

Figure 3. SEM images obtained from S2 samples of Capsul (part a and b) and Hi Cap 100 (part c and d) without

$(a$ and $c$ ) and with ( $b$ and $d$ ) the addition of $5 \%$ of vanilla extract. In all cases, the magnification was $1000 \times$

The numerical results of the diameters evaluation confirm that adding vanilla extract to the starches significantly decreases the average diameter of both Capsul and Hi Cap 100 particles obtained by spray drying. When adding $2.5 \%$ of VE, the average diameter of Capsul particles decreased from $8.3 \pm 5.5 \mu \mathrm{m}$ to $5.5 \pm 3.4 \mu \mathrm{m}$, as indicated by a t-test: $t(896)=9.3, \mathrm{p}<0.001$, whereas for Hi Cap 100 particles the diameters decreased from $6.5 \pm 3.4 \mu \mathrm{m}$ to $6.1 \pm 2.6 \mu \mathrm{m}$ for Hi Cap 100, also demonstrated with t-test: $t(921)=1.9, \mathrm{p}<0.05$. This effect becomes more pronounced when adding 5\% of vanilla extract: the average diameter of Capsul particles decreased from $8.3 \pm$ $5.5 \mu \mathrm{m}$ to $4.8 \pm 3.4 \mu \mathrm{m}$, and Hi Cap 100 from $6.5 \pm 3.4 \mu \mathrm{m}$ to $5.6 \pm 3.4 \mu \mathrm{m}$, as demonstrated with a t-test: $t(891)$ $=11.6, \mathrm{p}<0.001$ (for Capsul) and $t(443)=2.8, \mathrm{p}<0.005$ (for Hi Cap 100). On the other hand, the addition of the lowest proportion of vanilla extract (1\%) did not introduce significant changes in the average size of spray dried particles.

\subsection{Aroma Pattern, Encapsulation Efficiency, and Flavor Release}

In Figure 4, two characteristic plots of e-nose measurements are shown. In part a, the (typical) increasing response signal for all 12 sensors (i.e., one line for each sensor) is displayed against the time of measurement, in this case for the aroma released from Hi Cap 100 with $2.5 \%$ of VE in the stage of re-suspension (S3) after a spray-drying process.

In the radar plot displayed in part $\mathbf{b}$, for each sensor, the signal at the final time point (i.e., second 1200) is presented for the S1, S2, and S3 samples (i.e., S1 = the initial slurry, S2 = the powder obtained after drying, and $\mathrm{S} 3=$ the re-suspension of the powder in water to get the same initial starch concentration as in S1 samples). The whole process of obtaining S1, S2, and S3 samples is outlined in the scheme placed at the left of the radar plot. 
a

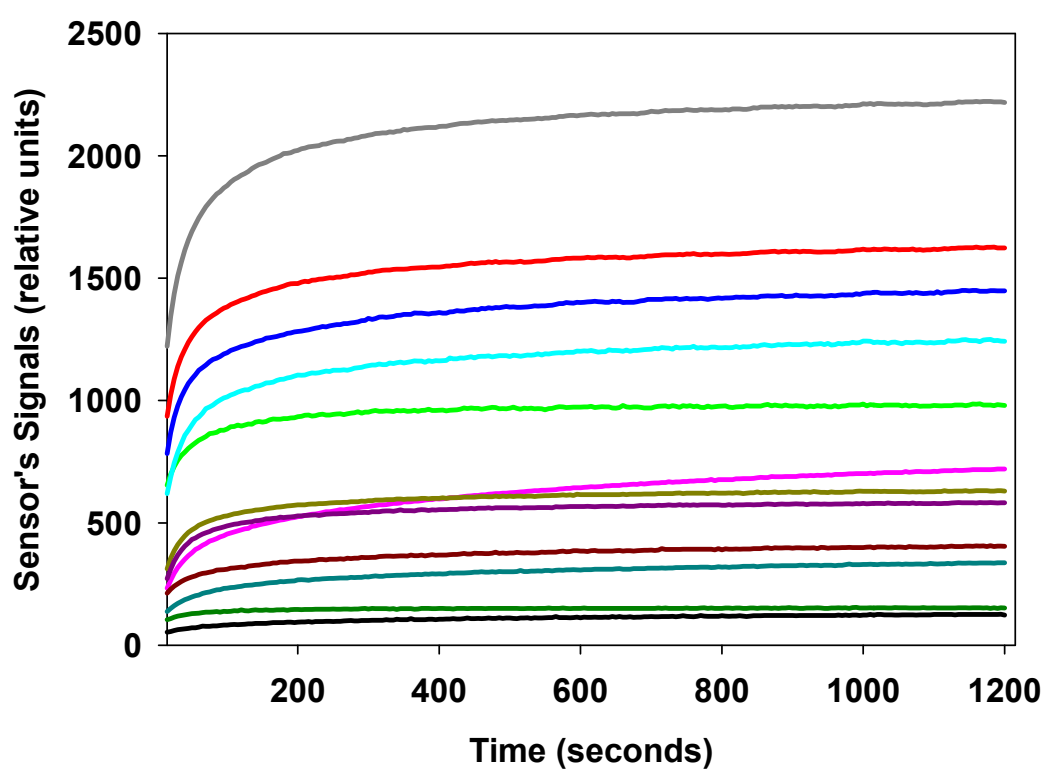

b

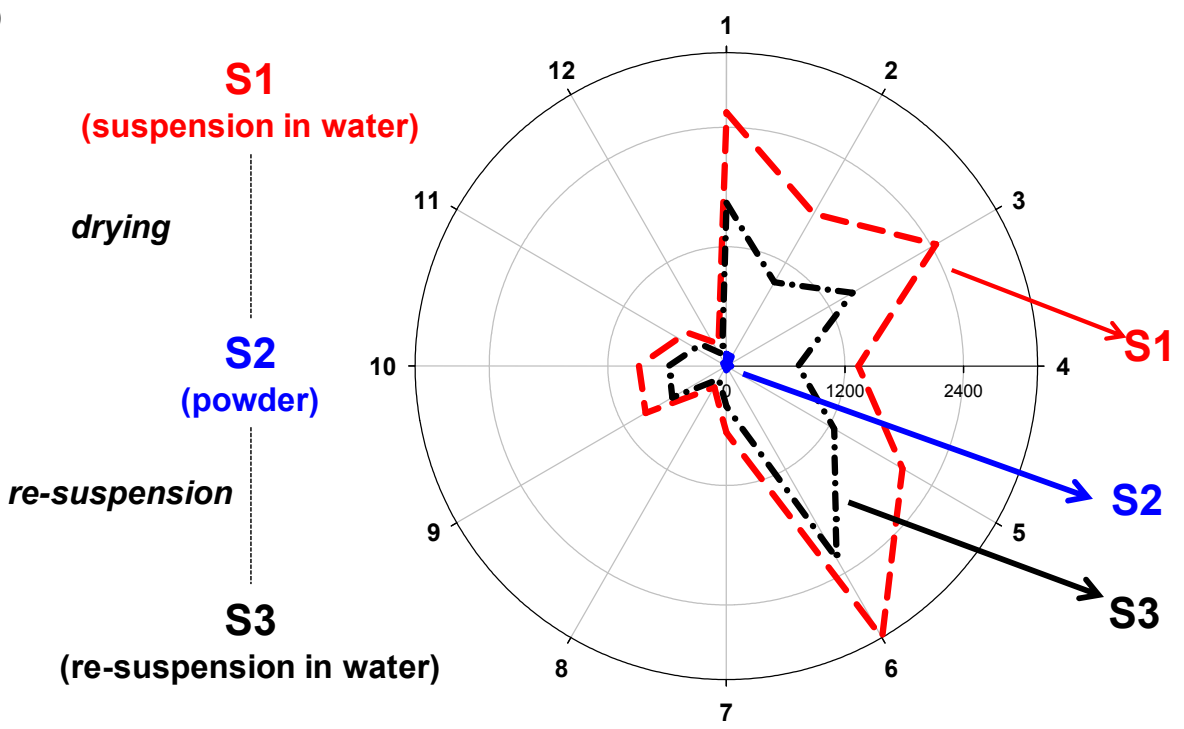

Figure 4. (a) Typical e-nose measurements for a spray-dried sample of Hi Cap 100 with $2.5 \%$ of vanilla extract. Each line represents the increasing signal of a single sensor over time. (b) Radar plot of the sensor signal of the last measurement time point for each sensor (numbered from 1 to 12) for a S1 (red dashed line), S2 (blue solid line), and S3 (black dotted-dashed line) sample of a Hi Cap 100 starch with $2.5 \%$ of vanilla extract along the spray-drying process

The S1 samples of this radar plot have been flavored with $2.5 \%$ of vanilla extract (see section 2.2.1). In this plot, one can clearly see that, for all sensors, the largest signal was recorded for the S1 sample. When this S1 sample is dried, becoming the S2 sample (powder), the signals of all sensors decreased dramatically, encircling a very small area near the origin (pointed by the blue arrow). The S3 sample, obtained by re-suspension of the S2 sample in water, recovered a significant portion of the initial intensity of the sensor's signals, resulting in a radar plot with equal shape to the one of the radar plot for the S1 sample.

It is important to remark again that the shape of the $\mathrm{S} 1$ and $\mathrm{S} 3$ radar plots in Figure $4 \mathrm{~b}$ are extremely similar, the only difference being the intensity of the signals. In particular, the relative units of the signal of each sensor are 
lower for the S3 samples (i.e., re-suspension of the S2 powder) than for the original suspension S1. For example, for sensor number 3, the signal for S1 has a value around 2400 whereas for S3 the value lowers down to about 1300. Note that the natural flavor contains about $90 \%$ of pure vanillin (4-hydroxy-3-methoxybenzaldehyde) and only a small percentage (traces) of other components (Bettazzi et al., 2006). Because the extract has one main component in a very high ratio with respect to the others, the radar plot changes only the area/intensity but not the shape. A detailed analysis concerning the relationship between shape and intensity was previously discussed (Rodríguez et al., 2010).

To confirm that the lower sensor's signals for the S3 samples are due to a partial loss of flavor during the drying process, the flavor encapsulated in the S2 powder was extracted during one and a half hour with ethanol and the amount of vanilla encapsulated was determined spectrophotometrically. The methodology is described in detail in section 2.2.4. The results showed that Capsul (Hi Cap 100) encapsulated $64 \%(56 \%)$ and $42 \%(39 \%)$ of the initially added vanilla when using spray and freeze drying, respectively, confirming that a part of the flavor is lost during both drying processes.

In order to investigate whether a molecular interaction between the starch and the vanilla takes place in the first slurry (S1), resulting in the initial aroma being differentially affected depending on the used starch, the raw e-nose data were analyzed by means of PCA and CA on the sample by sensor data, with only taking information regarding the last measurement (i.e., second 1,200) into account. The results showed that $\mathrm{S} 1$ samples cannot be discriminated by the type of starch used. The two starch types got mixed up in the component plot and a cluster analysis with two clusters resulted in $38 \%$ of misclassifications (i.e., $38 \%$ of the samples were assigned to the wrong starch type cluster). Next, in order to determine whether including information about earlier time points would allow us to better discriminate among the starches in the first suspension stage, we repeated the analysis using the sample by sensor by time point data. First, applying unfolded PCA to the three-way data resulted in a better resolution than applying PCA to the two-way data. However, Capsul and Hi Cap 100 S1 samples still got mixed up. Next, in order to corroborate the results obtained with PCA and U-PCA, we applied a PARAFAC (Harshman, 1970) and a PARAFAC2 (Harshman, 1972) analysis to the three-way data. The reason for doing this was to obtain a more adequate, robust, and interpretable model (Bro, 1997). Nonetheless, applying PARAFAC and PARAFAC2 (with two components) to the three-way data did not result in a clear discrimination of the S1 samples into the two starch types.

At the contrary, analyzing the S3 samples (i.e., re-suspension after drying) with PCA (with two components) and CA (with two clusters) resulted in a clear separation of the samples into two groups which correspond to the freeze and spray dried samples. In particular, in the component plot presented in Figure 5, the two separated groups of samples, which are surrounded by ellipses, can be clearly seen. Moreover, as can be seen in Figure 5, both within the freeze and spray dried group of samples, the two starch types can be clearly distinguished. In particular, applying a CA with 4 clusters resulted in a perfect classification of the samples into the four sample types (Capsul spray dried, Hi Cap 100 spray dried, Capsul freeze dried, and Hi Cap 100 freeze dried). Further, it appears that the differences between Hi Cap 100 and Capsul starches are more subtle when freeze-drying has been adopted than when spray drying has been used. Note that although the first component explains almost all the variance in the data, the addition of the second and third components, as can be seen in Figure 5, clearly improves the (visual) discrimination of the four sample types. The reason for this is that the components that explain the most variation in the data are not necessarily the components that best discriminate the samples, resulting in less important components (i.e., that do not explain much variance) sometimes being needed to discriminate the samples well (for more information, see Vicki \& Kiers, 2001). 


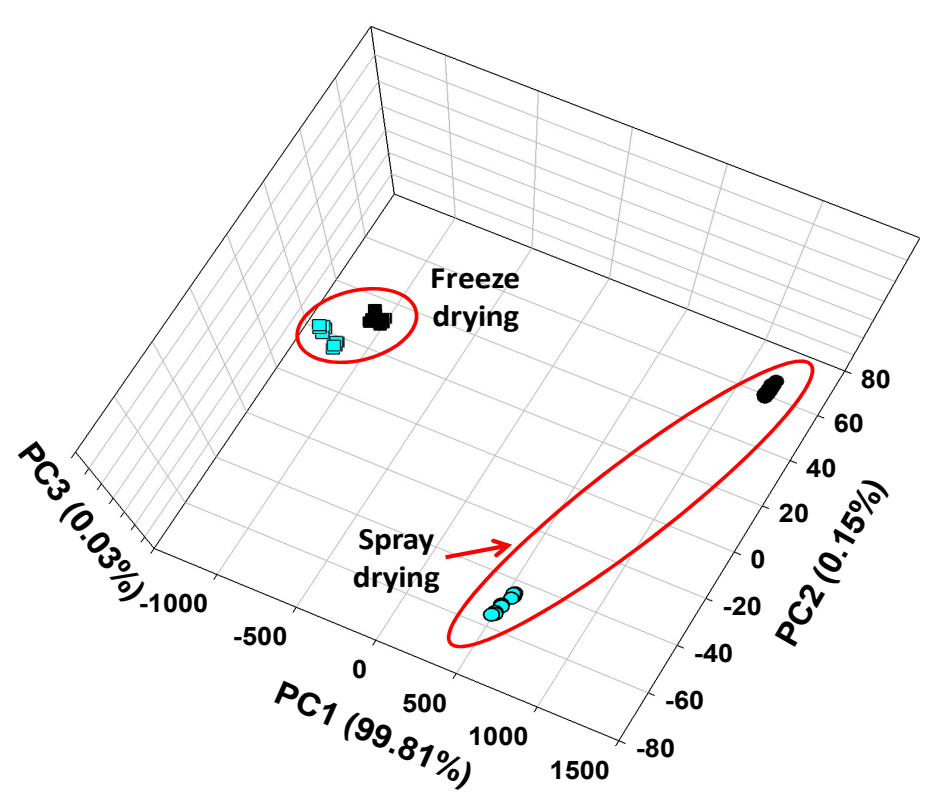

Figure 5. Plot of the sample scores on the first three components (PC1, PC2, and PC3) obtained from a (principal axis) PCA analysis of the sensor signals (last second) of the $\mathrm{S} 3$ samples (i.e., re-suspension), which are indicated as follows: Freeze dried Capsul (₫); Freeze dried Hi Cap $100(\square)$; Spray dried Capsul (•); Spray dried Hi Cap

$100(\bullet)$. The amount of variance that is explained by each component is indicated between parentheses

\section{Discussion}

The present study points out that valuable information can be obtained about the development of the molecular interaction between vanilla extract and OSA starches by means of GLCM texture analysis of SEM images and sensor array detection of the aroma released by the samples.

The information obtained by the SEM images shows that freeze drying yields powders without significant differences among Capsul and Hi Cap 100, while the spray drying method produces particles with textural features that clearly differ among both starch types. The SEM images for both starches show that most of the particles exhibit concavities on the surface. These concavities, which are called dents, are produced by a phenomenon known as surface indentation. This phenomenon has been frequently observed with wall materials consisting of polysaccharides. The formation in the particles has been attributed to atomization and drying parameters, uneven shrinkage at early stages of drying, and to the effect of a surface tension-driven viscous flow (Sheu \& Rosenberg, 1998).

The texture analysis of SEM images yields additional information in that the GLCM parameters clearly show that, for spray dried samples, Capsul yields rougher particles than Hi Cap 100. In Table 1, the contrast parameter $\mathrm{CT}$ is the most sensitive parameter for changes in roughness. Moreover, the CT parameter indicates that the addition of vanilla contributed to modify (reduce) the roughness in both Capsul and Hi Cap 100 particles. These differences, however, were not significant, at least not for the number of samples that were tested in this work. Furthermore, the addition of flavor results in, for both starch types, the average size of the particles being significantly decreased when compared to control samples (see Figure 3).

The use of a gas sensor array (e-nose) provided additional and complementary information, which considerably enriched the overall analysis. The three consecutive stages that a sample went through during the whole encapsulation process revealed different stages of the interaction between the flavor and the starches which can be easily discriminated. When analyzing the sensor readings by a multivariate data analysis method, no differences in headspace aroma released by the two starch types were found in the initial starch plus extract S1 slurry. This indicates that for the two particular starch types that were used in this study, the flavor does not interact closely with the starch in a way that allows detecting a change in the aroma.

Thus, the results clearly show that the real interaction between vanilla and the starch occurs when samples are dried, either by freeze or spray drying. This is reasonable since during the drying steps, the loss of the hydration 
of sample's components allows the intimate molecular contact which is needed for the adsorption process to occur, favored in this case by the OSA modification which enhances starch hydrophobicity (Cova et al., 2010).

Additionally, the e-nose data show that drying processes with the OSA modified starches produce powders which efficiently trap the flavor, as demonstrated by the sharp decrease of all sensors' signals for S2 samples (see Figure 4b) which is irrespective of the starch type and the used drying method. As expected, little or no measurable volatiles are detected by the e-nose, due to the vanillin (and the other trace components) low vapor pressures.

Re-suspension of the S2 samples in water (i.e., S3 samples) results in the aroma being released again. As a consequence, the radar plots for both samples have exactly the same shape, however with a lower intensity for the S3 samples, which can be explained by the loss of flavor during the drying processes. This is confirmed by an extraction and a spectrophotometric quantification of vanillin, which indicate that the spray drying methodology yields a more efficient vanilla encapsulation than freeze drying. Moreover, Capsul is more efficient than Hi Cap 100 for vanilla encapsulation. The latter difference among both starch types is also reflected in that, for spray dried samples, Capsul yields higher aroma signals than Hi Cap 100, that is, all sensor signals of Capsul S3 samples are higher than those obtained with Hi Cap 100 in matching conditions.

These results are in line with the evidence obtained from the textural parameters extracted by GLCM (see Table 1), which demonstrate that Capsul particles are much rougher than Hi Cap 100 particles. In this regard, previous reports also indicate that a higher surface area favors larger flavor retention with different types of starches (Boutboul et al., 2002).

It would be an interesting topic for further study to vary wall composition and drying parameters with the aim of determining the optimal operating conditions for these two OSA starches, and to evaluate thereafter its influence in the encapsulation efficiency of flavors and the modulation of the released aroma.

\section{Conclusions}

We compared the encapsulation performance of vanilla extract by two different OSA modified starches Capsul and Hi Cap 100 by spray and freeze drying procedures. The results clearly state that Capsul has a better capacity for vanilla encapsulation than Hi Cap 100. The combination of GLCM image texture analysis and e-nose measurements allowed us to demonstrate that the efficiency of the encapsulation and the recovery of the final aroma depend on the texture properties produced by a combination of the starch structure and the chosen drying process.

Furthermore, this work describes a useful and systematic tool that may stimulate new studies about the molecular interactions of scent molecules and carbohydrates, with the expectation of the aroma release being modulated by manipulating the drying process and the molecular structure of the starch.

\section{Acknowledgements}

D.L.B. is an Independent Researcher at the Consejo Nacional de Investigaciones Cientificas y Tecnológicas (CONICET, Argentina). S.D.R. is a recipient of a fellowship from CONICET. T.F.W. is a post-doctoral researcher at the Fund for Scientific Research (FWO)-Flanders. This work has been supported by CONICET (PIP 6382 and PIP 01210). The authors are grateful to Gelfix S.A. (Argentina) for providing the starch samples.

\section{References}

Andersson, C. A., \& Bro, R. (2000). The N-way Toolbox for MATLAB. Chemometrics and Intelligent Laboratory Systems, 52(1), 1-4. http://dx.doi.org/10.1016/S0169-7439(00)00071-X

Anwar, S. H., \& Kunz, B. (2011). The influence of drying methods on the stabilization of fish oil microcapsules: Comparison of spray granulation, spray drying, and freeze drying. Journal of Food Engineering, 105(2), 367-378. http://dx.doi.org/10.1016/j.jfoodeng.2011.02.047

Arzate-Vázquez, I., Chanona-Pérez, J. J., Calderón-Domínguez, G., Terres-Rojas, E., Garibay-Febles, V., Martínez-Rivas, A., \& Gutiérrez-López, G. F. (2012). Microstructural characterization of chitosan and alginate films by microscopy techniques and texture image analysis. Carbohydrate Polymers, 87(1), 289-299. http://dx.doi.org/10.1016/j.carbpol.2011.07.044

Bettazzi, F., Palchetti, I., Sisalli, S., \& Mascini, M. (2006). A disposable electrochemical sensor for vanillin detection. Analytica Chimica Acta, 555(1), 134-138. http://dx.doi.org/10.1016/j.aca.2005.08.069

Bhosale, R., \& Singhal, R. (2006). Process optimization for the synthesis of octenyl succinyl derivative of waxy corn and amaranth starches. Carbohydrate Polymers, 66(4), 521-527. 
http://dx.doi.org/10.1016/j.carbpol.2006.04.007

Boutboul, A., Giampaoli, P., Feigenbaum, A., \& Ducruet, V. (2002). Influence of the nature and treatment of $\begin{array}{lllll}\text { starch on aroma retention. } & \text { Carbohydrate Polymers, }\end{array}$ http://dx.doi.org/10.1016/S0144-8617(01)00160-6

Bro, R. (1997). PARAFAC. Tutorial and applications. Chemometrics and Intelligent Laboratory Systems, 38, 149-171. http://dx.doi.org/10.1016/S0169-7439(97)00032-4

Carroll, J. D., \& Chang, J. (1970). Analysis of individual differences in multidimensional scaling via an N-way generalization of "Eckart-Young" decomposition. Psychometrika, 35, 283-319. http://dx.doi.org/10.1007/BF02310791

Cova, A., Sandoval, A. J., Balsamo, V., \& Müller, A. J. (2010). The effect of hydrophobic modifications on the adsorption isotherms of cassava starch. Carbohydrate Polymers, 81(3), 660-667. http://dx.doi.org/10.1016/j.carbpol.2010.03.028

Gharsallaoui, A., Roudaut, G., Chambin, O., Voilley, A., \& Saurel, R. (2007). Applications of spray-drying in microencapsulation of food ingredients: An overview. Food Research International, 40(9), 1107-1121. http://dx.doi.org/10.1016/j.foodres.2007.07.004

Haddi, A., Amari, A., Alami, H., El Bari, N., Llobet, E., \& Bouchikhi, B. (2011). A portable electronic nose system for the identification of cannabis-based drugs. Sensors and Actuators B: Chemical, 155(2), 456-463. http://dx.doi.org/10.1016/j.snb.2010.12.047

Harshman, R. A. (1970). Foundations of the PARAFAC procedure: Models and conditions for an "explanatory" multi-modal factor analysis. UCLA Working Papers in Phonetics, 16, 1-84.

Harshman, R. A. (1972). PARAFAC2: Mathematical and technical notes. UCLA Working Papers in Phonetics, $22,30-44$.

Johnson, R. A., \& Wichern, D. W. (2002). Applied multivariate statistical analysis. New Jersey: Prentice Hall.

Jolliffe, I. T. (2002). Principal Component Analysis (2nd ed.). New York: Springer.

Kamruzzaman, M., El-Masry, G., Sun, D.-W., \& Allen, P. (2012). Prediction of some quality attributes of lamb meat using near-infrared hyperspectral imaging and multivariate analysis. Analytica Chimica Acta, 714, 57-67. http://dx.doi.org/10.1016/j.aca.2011.11.037

Kiers, H. A. L. (2000). Towards a standardized notation and terminology in multiway analysis. Journal of Chemometrics, 14(3), 105-122.

Lopez-Quiroga, E., Antelo, L. T., \& Alonso, A. A. (2012). Time-scale modeling and optimal control of freeze-drying. Journal of Food Engineering, 111, 655-666. http://dx.doi.org/10.1016/j.jfoodeng.2012.03.001

Nomikos, P., \& MacGregor, J. F. (1994). Monitoring batch processes using multiway principal component analysis. AIChE Journal, 40(8), 1361-1375. http://dx.doi.org/10.1002/aic.690400809

Peris, M., \& Escuder-Gilabert, L. (2009). A 21st century technique for food control: Electronic noses. Analytica Chimica Acta, 638(1), 1-15. http://dx.doi.org/10.1016/j.aca.2009.02.009

Pisano, R., Fissore, D., \& Barresi, A. A. (2011). Freeze-drying cycle optimization using model predictive control techniques. Industrial \& Engineering Chemistry Research, 50(12), 7363-7379. http://dx.doi.org/10.1021/ie101955a

Ratchahat, S., Viriya-empikul, N., Faungnawakij, K., Charinpanitkul, T., \& Soottitantawat, A. (2010). Synthesis of carbon microspheres from starch by hydrothermal process. Science Journal Ubon Ratchathani University, $1(2), 40-45$.

Rodriguez, S. D., Monge, M. E., Olivieri, A. C., Negri, R. M., \& Bernik, D. L. (2010). Time dependence of the aroma pattern emitted by an encapsulated essence studied by means of electronic noses and chemometric analysis. Food Research International, 43(3), 797-804. http://dx.doi.org/10.1016/j. foodres.2009.11.022

Sheu, T. Y., \& Rosenberg, M. (1998). Microstructure of microcapsules consisting of whey proteins and $\begin{array}{lllll}\text { carbohydrates. Journal of Food } & \text { 491-494. }\end{array}$ http://dx.doi.org/10.1111/j.1365-2621.1998.tb15770.x

Skov, T., \& Bro, R. (2005). A new approach for modelling sensor based data. Sensors and Actuators B: Chemical, 106(2), 719-729. http://dx.doi.org/10.1016/j.snb.2004.09.023 
Tai, A., Sawano, T., Yazama, F., \& Ito, H. (2011). Evaluation of antioxidant activity of vanillin by using multiple antioxidant assays. Biochimica et Biophysica Acta (BBA) General Subjects, 1810(2), 170-177. http://dx.doi.org/10.1016/j.bbagen.2010.11.004

Tournier, C., Grass, M., Zope, D., Salles, C., \& Bertrand, D. (2012). Characterization of bread breakdown during mastication by image texture analysis. Journal of Food Engineering, 113(4), 615-622. http://dx.doi.org/10.1016/j.jfoodeng.2012.07.015

Vichi, M., \& Kiers, H. A. L. (2001). Factorial k-means analysis for two-way data. Computational Statistics and Data Analysis, 37, 49-64. http://dx.doi.org/10.1016/S0167-9473(00)00064-5

$\mathrm{Yu}, \mathrm{X}$. Liu, K., Wu, D., \& He, Y. (2012). Raisin quality classification using least squares support vector machine (LSSVM) based on combined color and texture features. Food and Bioprocess Technology, 5(5), 1552-1563. http://dx.doi.org/10.1007/s11947-011-0531-9

Zheng, C., Sun, D.-W., \& Zheng, L. (2006). Recent applications of image texture for evaluation of food qualities - a review. Trends in Food Science \& Technology, 17(3), 113-128. http://dx.doi.org/10.1016/j.tifs.2005.11.006 